\title{
Characterizing the influence of parent grain structures on the physical properties of additively manufactured Ti-64 alloys using EBSD
}

Pat Trimby ${ }^{1}$, Iain Anderson ${ }^{2}$, Klaus Mehnert ${ }^{3}$, John Porter ${ }^{4}$ and John Wheeler ${ }^{5}$

${ }^{1}$ Oxford Instruments, High Wycombe, England, United Kingdom, ${ }^{2}$ Oxford Instruments NanoAnalysis, United States, ${ }^{3} \mathrm{ST}$ Development GmbH, Paderborn, Germany, ${ }^{4}$ Castheon Inc., Thousand Oaks, California, United States, ${ }^{5}$ The University of Liverpool, Liverpool, England, United Kingdom

Ti6A14V alloy, known as Ti64, is a two-phase titanium alloy with ideal properties for the aerospace industry, including high strength and low density [1]. In addition, Ti64's excellent corrosion resistance and biocompatibility makes it an attractive alloy for use in medical implants. Conventional manufacturing of Ti64 products is challenging due to its poor thermal conductivity and high levels of strain hardening, resulting in high costs and high wastage. Additive manufacturing (AM), in comparison, enables much more efficient manufacture of Ti64 components with significantly less waste and with an increased capability to fabricate complex shapes. However, AM processes such as electron powder bed fusion (ePBF) and laser powder bed fusion (L-PBF) involve rapid melting of powder-based feedstock and subsequent resolidification and cooling. The resulting large temperature gradients and phase transformation from the high-temperature $\beta$-Ti phase to the low-temperature $\alpha$-Ti phase can lead to high residual stresses and defects within AM built Ti64 components, especially when there is no powder bed heating, with a detrimental effect on the material's final mechanical properties.

It is well established that additively manufactured Ti64 grows from the melt with cubic $\square$-grains building epitaxially on underlying $\beta$-grains, typically with a strong $<100>$ texture aligned with the build direction. On cooling the $\beta$-microstructure largely transforms to the hexagonal $\alpha$-phase with 12 possible crystallographic variants (as defined by the Burgers orientation relationship: $\{110\}_{\beta} \|\{0001\}_{\alpha}$ and $\left.\langle-11-1\rangle_{\beta} \|<2-1-10\right\rangle_{\alpha}$ ): therefore, the grain size and crystallographic orientation of the $\beta$ microstructure has a significant influence on the resulting $\alpha$-microstructure and the material's subsequent physical properties. Although the reconstruction of the parent $\beta$-grain structure from the daughter $\alpha$-grain structure is not trivial, it is possible to use orientation data collected using electron backscatter diffraction (EBSD) to determine the parent grain orientations from the daughter variant grain structure. This process has been implemented in the AZtecCrystal EBSD data processing software, using the orientation relationship refinement and voting scheme as proposed by Huang et al. [2]. This, coupled with the latest high speed CMOS EBSD detectors, enables rapid analysis and subsequent reconstruction of the original $\beta$-microstructure and microtexture in additively manufactured Ti64 alloys. EBSD data can also be processed to determine the bulk elastic properties of the sample, as well as to reveal information about the nature and density of geometrically necessary dislocations [3]. This allows us to relate the original high temperature $\beta$-grain structure and texture with the final elastic and deformation properties of the as-built component.

Here we show data from a series of Ti64 alloys that have been manufactured using an Arcam ePBF process, with subsequent hot isostatic pressing to reduce porosity. The samples were subjected to tensile test experiments, prior to preparation for EBSD analyses. The EBSD scans were carried out at a range of scales, from whole sample analyses (field of view $\sim 15 \mathrm{~mm}$ ) to higher resolution scans (field of view 1-2 $\mathrm{mm})$. The prior $\beta$-microstructure was reconstructed, as shown by the example in Figure 1, enabling grains with a strong $<100>$ texture to be separated from grains with different textures. The characteristics of the $\alpha$-microstructures that had derived from $\beta$-grains with specific textures were then 
examined in detail, in particular to determine the nature of the geometrically necessary dislocations, the dominant slip systems and the elastic properties of the final samples.

A typical example is shown in Figure 2: here the parent $\beta$-microstructure for the whole sample has been reconstructed, showing the dominant expected $\langle 100>$ texture parallel to the build direction (horizontal in the map). The Young's Modulus values of the final $\alpha$-microstructure have been plotted for areas derived from $\beta$-grains with this $<100>$ texture and compared to areas derived from $\beta$-grains with a strong $<110>$ texture (green in the map): the results show how the parent grain orientation plays a significant role in determining the elastic properties of the final Ti64 microstructure, with a higher Young's Modulus (for loading parallel to the build direction) for regions that were derived from $\beta$ grains with a strong $<110>$ texture. Similar contrasts were observed in the nature of dislocations between these different areas, with the dominant slip system shown to be closely linked to the parent grain texture.

This study shows the value of being able to reconstruct the high temperature parent grain microstructure in additively manufactured Ti64 alloys: using rapid EBSD analysis and in-depth data processing tools, we can show that the final mechanical properties of these Ti64 samples are directly linked to the texture of the original high temperature $\beta$-microstructure.

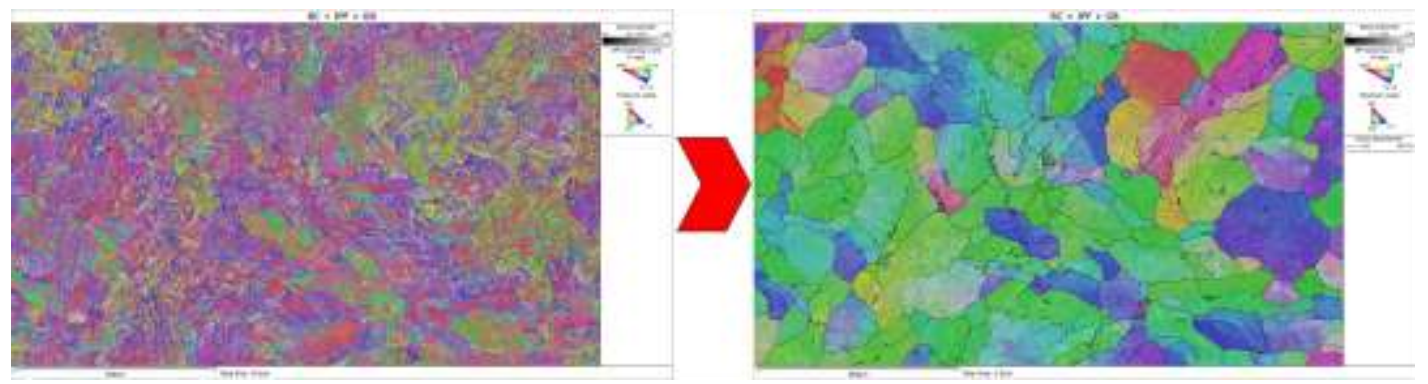

Figure 1. EBSD orientation maps (colored using the inverse pole figure coloring scheme, parallel to the build direction / surface normal) showing the parent $\beta$-grain structure (right) derived from the asmeasured $\alpha$-grain structure (left) in an additively manufactured Ti64 sample. Field of view $1.5 \mathrm{~mm}$ wide.

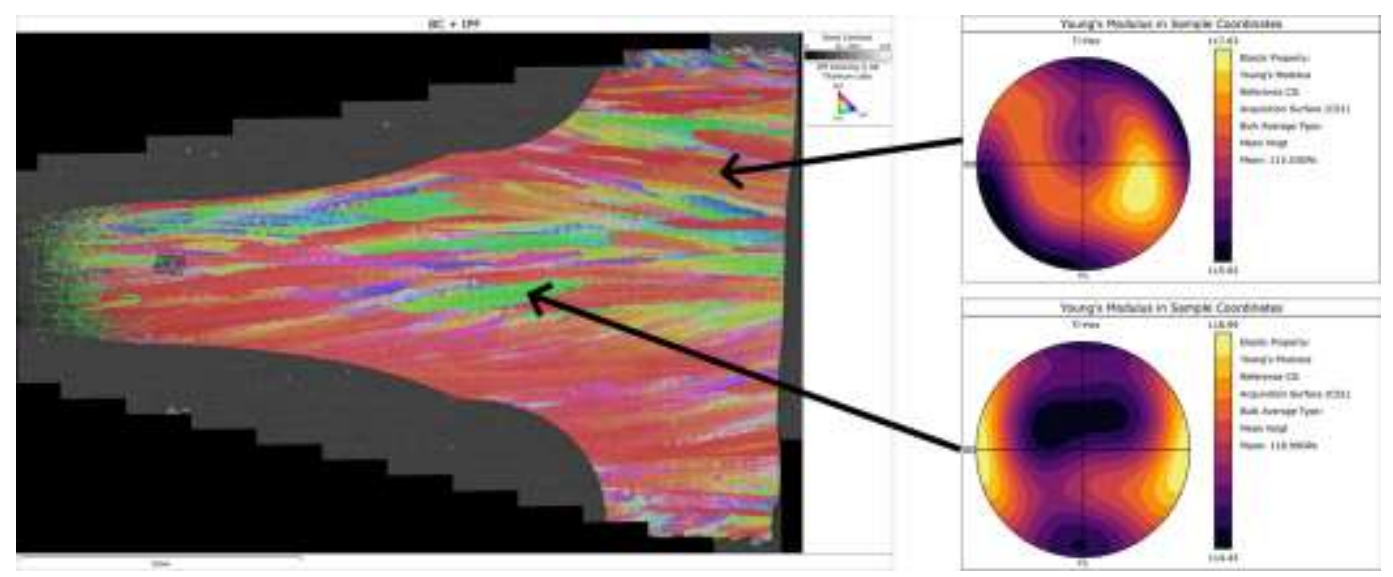

Figure 2. EBSD orientation map showing the reconstructed parent $\beta$-grain structure of a whole Ti64 tensile test sample (field of view $14 \mathrm{~mm}$ across), colored using the inverse pole figure coloring scheme, 
parallel to the build direction / map x-direction. To the right are plots showing the Young's Modulus variations for the $\alpha$-grains that have derived from prior $\beta$-grains with a strong texture (red colored grains) at the top, and from prior $\beta$-grains with a strong texture (green colored grains) at the bottom. Note the higher Young's modulus for loading parallel to the build direction (BD) in microstructures derived from the textured grains.

\section{References}

1. S. Liu and Y.C. Shin, Materials and Design, 164 (2019) 107552

2. C.-Y. Huang et al., Materialia, 9 (2020) 100554

3. J. Wheeler et al., J. Microscopy, 233 (2009) pp. 482-494 